\title{
Development of Focal segmental glomerulosclerosis 4- years following direct- acting antiviral therapy for hepatitis $C$ virus infection: Case report
}

\author{
Abdelmoneim Elhadidy ${ }^{1, *}$, Rania Maher ${ }^{2}$, Ahmed Ehab $^{2}$. \\ ${ }^{1}$ Damietta Fever and Gastroentrology Hospital, Egypt, ${ }^{2}$ Hepatology and Gastroentrology Unit, Damietta Cardiology and \\ Gastroenterology Center, Damietta, Egypt.
}

\begin{abstract}
It is well celebrated that, eradication of HCV RNA from serum is commonly accompanied by clinical improvement of glomerular abnormalities. Sofosbuvir (SOF) is the only commonly used direct-acting antiviral therapies (DAAs) that is primarily cleared by kidney. The effect of sustained virologic response (SVR) on renal function is wellestablished in the setting of interferon (IFN) based regimens. Patients who achieved SVR with IFN regimens have a decreased incidence of chronic kidney disease and endstage renal disease. However this effect following DAAs therapy is scarce. There is no clinically relevant change in renal function among the majority of $\mathrm{HCV}$ infected patients following completion of DAA-based treatments. During follow up of patients with SVR following DAAs therapy, we observed one patient has 4 years SVR post DAAs (Sofosbuvir plus dacklatavir) who developed resistant edema and ascites with massive proteinuria following treatment with DAAs therapies and whose kidney biopsies demonstrated focal segmental glomerulosclerosis (FSGS) hilar type and interstitial fibrosis and tubular atrophy.
\end{abstract}

\section{Introduction}

Direct-acting antiviral agents are now the main option therapy for chronic HCV infection with an excellent safety profile and promising clinical outcomes 1,2 . There is relatively infrequent evidence suggesting DAA treatment may improve renal function ${ }^{3}$. Focal segmental glomerulosclerosis (FSGS) is a histologically renal injury characterized by sclerosis, hyalinosis, foam-cell infiltration, vacuolization of podocytes, and podocyte precursor proliferation. FSCS affects only some glomeruli and only a portion of the affected glomerulus is sclerosed ${ }^{4}$. Idiopathic FSGS is the commonest primary glomerular histology in the United States, and one of the principal causes of idiopathic nephrotic syndrome in adults, a main prognostic factor in the progression to end-stage renal disease (ESRD) ${ }^{5}$.

Keywords: Focal segmental glomerulosclerosis, hepatitis $\mathrm{C}$ virus infection, direct-acting antiviral therapies, sustained virologic response.

Received: 4-9-2021; Accepted: 7-10-2021

* Corresponding author. email: abdelmoneimelhadidy@yahoo.com
Previous study has shown a slight decline of estimated glomerular filtration rate (eGFR) at end of treatment of DAAs, followed by a slight rise 12 weeks after treatment. However, long-term effect of nephron- toxicity exerted by DAAs has not been well cleared ${ }^{6}$. In this report, we describe a case of a severe focal segmental glomerulosclerosis 4 years post - sustained virologic response following HCV treatment with DAA in an HCVinfected patient.

\section{Case Presentations}

A 47 years old male with no history of renal impairment, not diabetic not hypertensive received DAAs and has SVR. After 4 years-post-SVR, during follow up, the patient presented by refractory edema and abdominal enlargement. Abdominal ultrasound reveled, average size normal liver echopattern; normal size and shape of spleen; normal size, site, shape and echopattern of both kidneys and moderate ascites. The patient has F 0/Fibroscane. Laboratory investigation showed that, ALT (30 U/L), AST (25U/L), serum bilirubin $(0.9 \mathrm{mg} / \mathrm{dl})$, serum albumin was (1.8 g/dl), AFP (1.7 IU/ml), WBC $5.3\left(10^{3} / \mathrm{cmm}\right), \mathrm{Hg} 10$ $\mathrm{g} / \mathrm{dl}$, platelets $174\left(10^{3} / \mathrm{cmm}\right)$ and serum creatinine $(3.5$ $\mathrm{mg} / \mathrm{dl})$.

HIV negative, antimitochondrial antibodies (AMA) and anti-liver-kidney microsomal (ALKM) antibody were negative. Normal cardiological examination by consultation. Diagnostic paracentesis was done and revealed, ascitic protein was $2.2 \mathrm{gm} / \mathrm{dl}$, WBC 100 $\left(10^{3} / \mathrm{cmm}\right)$ and SAG was less than 1.1 suggested noncirrhotic ascites. Urinalysis showed massive protein in urine in 24/ $\mathrm{Hrs}$ was $8.99 \mathrm{gm} / 24 \mathrm{hrs}$ (normal less than $0.15)$.

The patient was referred to nephrology department. The nephrologist suggested mostly 2ry nephrotic syndrome and advised renal biopsy. The result of pathological examination of renal tissue revealed (Focal segmental glomerulosclerosis hilar type (5/12 glom) and interstitial fibrosis and tubular atrophy about 25\% (Figure 1). The patient received corticosteroid $20 \mathrm{mg}$ twice day plus azathioprine $50 \mathrm{mg}$ twice day. Follow up after one month under treatment reveal improvement of the patient as serum albumin is $3.8 \mathrm{~g} / \mathrm{dl}$ and serum creatinine is 0.9 $\mathrm{mg} / \mathrm{dl}$ and abdominal ultrasonography no ascites. 
Discussion

Present evidence has confirmed that SVR may reduce the incidence of endstage renal disease and chronic kidney disease ${ }^{7}$. FSGS is a clinicopathologic diagnosis in patients who develop proteinuria due to podocyte injury and carries a poor renal prognosis ${ }^{8}$. It is different from $\mathrm{HCV}$ associated immune complex/cryoglobulinemic glomerulonephritis, which is caused by direct or indirect B-cell proliferation by $\mathrm{HCV}$, leading to pathogenic immune complex formation and deposition in glomerul ${ }^{9}$. In our case report, we found male patient with previously chronic HCV infection developed edema and ascites 4 years post sustained virologic response to DAAs therapy. DAAs may lead to a modest decline in eGFR. This decline in renal function tends to reverse after completion of treatment except with sofosbuvir-containing regimens ${ }^{6,10}$. However, the long-term clinical implications of this association are unclear.

Proposals for DAA therapy-associated FSGS may comprise direct podocyte toxicity and an immune reconstitution syndrome characterized by restoration of interferon tone during viral eradication ${ }^{11}$. We recommend routine urinalysis monitoring before and during DAA therapy and quick nephrology evaluation if proteinuria is spotted. Moreover, prospective clinical studies following up these patients for proteinuria and eGFR measurement are needed in this respect.

\section{References}

1. Abed, S., Mousa, N., and Soliman, R., et al. (2018). Recent trends in chronic hepatitis $C$ virus treatment. Medical Journal of Viral Hepatitis, 2(2) 1-6.

2. Dina, E., Nasser, M., Sarah, E., et al. (2020). Pretreatment serum alpha fetoprotein and its relation to sustained virologic response in patients with chronic HCV infection treated with directacting antiviral therapy. Medical Journal of Viral Hepatitis, 4(2), 69-73.

3. Goetsch, M. R., Tamhane, A., Varshney, M., et al. (2017). Direct-acting antivirals in kidney transplant patients: successful hepatitis C treatment and short-term reduction in urinary protein/creatinine ratios. Pathogens \& immunity, 2(3), 366.

4. Han, M. H., and Kim, Y. J. (2016). Practical application of Columbia classification for focal segmental glomerulosclerosis. BioMed research international, 2016.

5. Kitiyakara, C., Eggers, P., and Kopp, J. B. (2004). Twenty-one-year trend in ESRD due to focal segmental glomerulosclerosis in the United States. American Journal of Kidney Diseases, 44(5), 815-825.
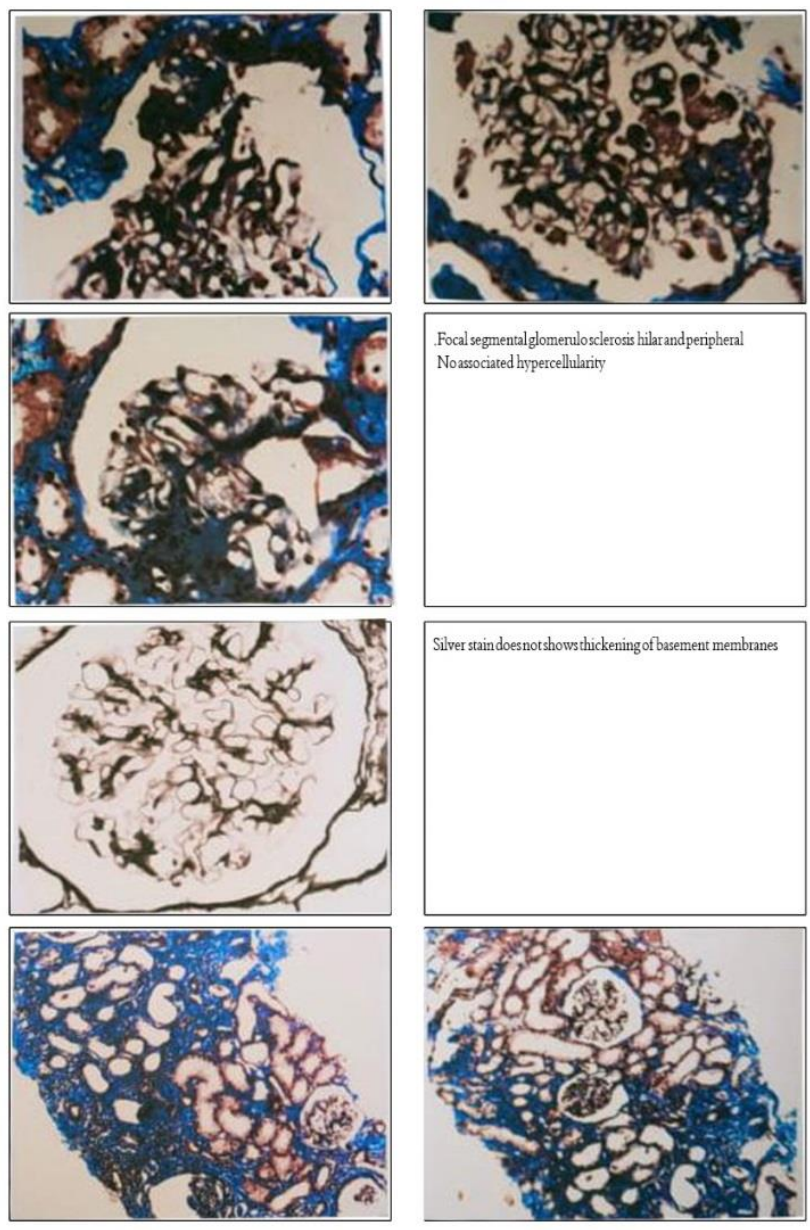

Figure 1. shows small areas of segmental sclerosis, hilar and peripheral, no associated hypercelluarity. Silver stain doesn't shows thickening of basement membrane. Interstitial fibrosis and tubular atrophy about $25 \%$

6. Tsai, M. C., Lin, C. Y., Hung, C. H., et al. (2019). Evolution of renal function under direct-acting antivirals treatment for chronic hepatitis C: A realworld experience. Journal of viral hepatitis, 26(12), 1404-1412.

7. Hsu, Y. C., Ho, H. J., Huang, Y. T., et al. (2015). Association between antiviral treatment and extrahepatic outcomes in patients with hepatitis $\mathrm{C}$ virus infection. Gut, 64(3), 495-503.

8. D'Agati, V. D., Kaskel, F. J., and Falk, R. J. (2011). Focal segmental glomerulosclerosis. New England Journal of Medicine,365(25), 23982411.

9. Fabrizi, F., Plaisier, E., Saadoun, D., et al. (2013). Hepatitis C virus infection, mixed cryoglobulinemia, and kidney disease. American Journal of Kidney Diseases, 61(4), 623-637. 


\section{Case Report}

10. Butt, A. A., Ren, Y., Puenpatom, A., et al. (2018). Effectiveness, treatment completion and safety of sofosbuvir/ledipasvirandparitaprevir/ritonavir/omb itasvirr+ dasabuvir in patients with chronic kidney disease: an ERCHIVES study. Alimentary pharmacology \& therapeutics, 48(1), 35-43.

11. Sise, M. E., Wisocky, J., Rosales, I. A., et al. (2016). Lupus-like immune complex-mediated glomerulonephritis in patients with hepatitis $\mathrm{C}$ virus infection treated with oral, interferon-free, direct-acting antiviral therapy. Kidney international reports, 1(3), 135-143. 